\title{
Following Molecular Mobility During Chemical Reactions: No Evidence for Active Propulsion
}

\author{
Lucy L. Fillbrook, ${ }^{\ddagger}, 1$ Jan-Philipp Günther, ${ }^{\ddagger}, 2,3$ Günter Majer, ${ }^{2}$ Daniel J. O’Leary, ${ }^{4}$ William S. Price, ${ }^{5}$ Hal \\ Van Ryswyk, ${ }^{6}$ Peer Fischer, ${ }^{*, 2,3}$ and Jonathon E. Beves.*,1 \\ ${ }^{1}$ School of Chemistry, UNSW Sydney, Sydney, NSW 2052, Australia. \\ ${ }^{2}$ Max Planck Institute for Intelligent Systems, Heisenbergstr. 3, 70569 Stuttgart, Germany. \\ ${ }^{3}$ Institute of Physical Chemistry, University of Stuttgart, Pfaffenwaldring 55, 70569 Stuttgart, Germany. \\ ${ }^{4}$ Department of Chemistry, Pomona College, 645 North College Ave, Claremont, CA 91711, USA. \\ ${ }^{5}$ Nanoscale Group, School of Science, Western Sydney University, Penrith, NSW 2751, Australia. \\ 6Department of Chemistry, Harvey Mudd College, 301 Platt Blvd, Claremont, CA 91711, USA. \\ KEYWORDS: enhanced diffusion, nuclear magnetic resonance, diffusion NMR, relaxation, copper-catalyzed click reaction.
}

\begin{abstract}
The reported changes in self-diffusion of small molecules during reactions have been attributed to "boosted mobility". We demonstrate the critical role of changing concentrations of paramagnetic ions on nuclear magnetic resonance (NMR) signal intensities, which lead to erroneous measurements of diffusion coefficients. We present simple methods to overcome this problem. The use of shuffled gradient amplitudes allows accurate diffusion NMR measurements, even with time-dependent relaxation rates caused by changing concentrations of paramagnetic ions. The addition of a paramagnetic relaxation agent allows accurate determination of both diffusion coefficients and reaction kinetics during a single experiment. We analyze a copper-catalyzed azide-alkyne cycloaddition 'click' reaction, for which boosted mobility has been claimed. With our methods, we accurately measure the diffusive behavior of the solvent, starting materials and product, and find no global increase in diffusion coefficients during the reaction. We overcome NMR signal overlap using an alternative reducing agent to improve the accuracy of the diffusion measurements. The alkyne reactant diffuses slower as the reaction proceeds, due to binding to the copper catalyst during the catalytic cycle. The formation of this intermediate was confirmed by complementary NMR techniques and density functional theory calculations. Our work calls into question recent claims that molecules actively propel or swim during reactions, and establishes that time-resolved diffusion NMR measurements can provide valuable insight into reaction mechanisms.
\end{abstract}

Motion at the molecular level ${ }^{1}$ is central for the development of molecular machines and devices. ${ }^{2}$ Biology is rich in examples of active movement in molecular systems, which provide inspiration for synthetic chemical analogues.1c,1d,1f The diffusive motion of molecular species can be monitored by dynamic light scattering, ${ }^{3}$ fluorescence correlation spectroscopy ${ }^{4}$ or nuclear magnetic resonance (NMR) spectroscopy. ${ }^{5}$ Diffusion NMR provides information on molecular motion and can be used to determine the size and diffusive properties of molecules and enzymes ${ }^{6}$ in situ and non-invasively. ${ }^{7}$

Diffusion NMR has been used to argue that molecular species involved in chemical reactions display mobility higher than passive Brownian motion. ${ }^{8}$ The energy released during the reaction was claimed to directly propel the molecule (displayed schematically in Figure 1a). ${ }^{8}$ Such a phenomenon would have tremendous implications for our fundamental understanding of chemical reactions. However, there is currently no theoretical explanation that supports these observations. ${ }^{1 f}$ The first example to claim enhanced diffusion of small molecules during a catalyzed reaction involved a Grubbs metathesis catalyst, ${ }^{9}$ although this was later found to be due to convection artefacts. ${ }^{10}$ More recently, it has been claimed that an increase in molecular mobility during reactions is a general phenomenon, as purportedly the reactants and the solvent show enhanced diffusion in a number of chemical reactions. ${ }^{8}$ In the most spectacular example, ${ }^{8} \mathrm{a}$ reactant in a copper-catalyzed azide-alkyne cycloaddition (CuAAC) 'click' reaction (Figure $1 \mathrm{~b}$ ) showed an apparent $60 \%$ increase in its diffusion coefficient, while the diffusion coefficient of the residual solvent had an apparent $20 \%$ increase. We re-examined this experiment and concluded that there is no enhanced diffusion, ${ }^{11}$ while the authors maintain their claims. ${ }^{12}$

Herein we outline factors that can affect diffusion NMR measurements of dynamic systems involving changing concentrations of paramagnetic species, which is the case for the $\mathrm{CuAAC}$ reaction. We show that the addition of an inert paramagnetic relaxation agent, in modest concentrations, can allow simultaneous measurement of accurate diffusion coefficients and kinetics, which is otherwise not possible. The results have been verified inde- 
pendently by three laboratories, using different types of NMR probes, acquisition methods and reagent sources.

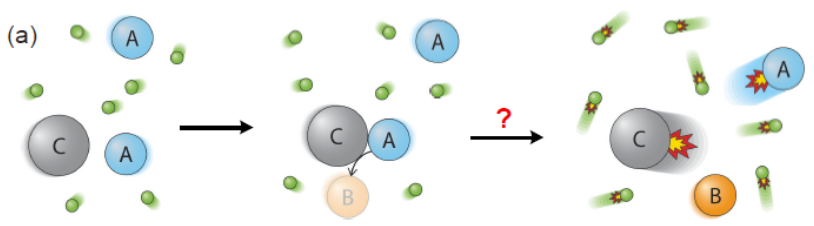

(b)(i) $\mathrm{Cu}^{2+}+$ ascorbate $\stackrel{-\mathrm{H}^{+}}{\longrightarrow} \mathrm{Cu}^{+}+$dehydroascorbate
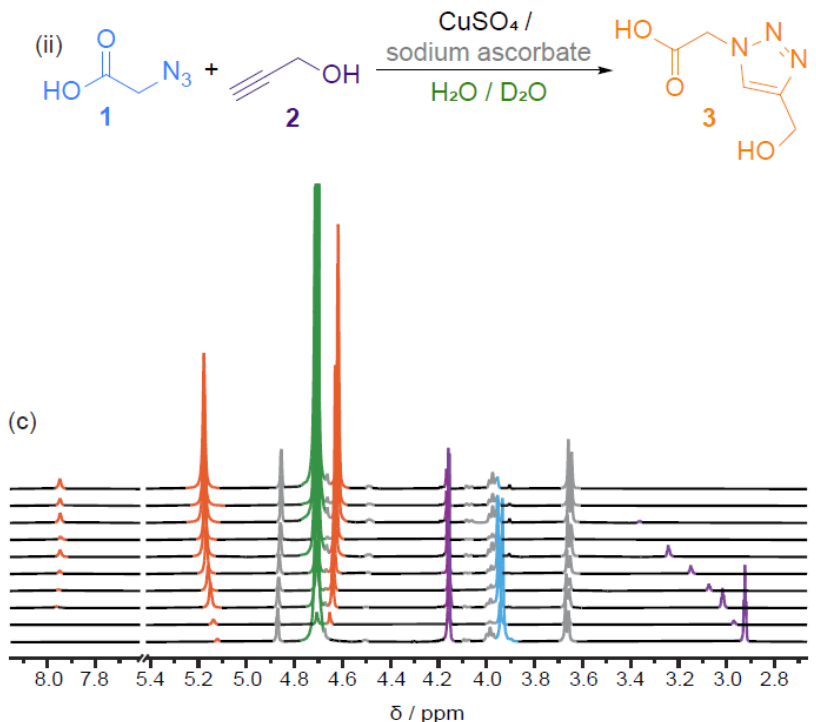

Figure 1. Enhanced diffusion during the CuACC reaction? (a) Proposed "boosted mobility": catalyst $\mathrm{C}$ converts species A to $\mathrm{B}$ while surrounded by solvent (green). The energy released during the reaction has been claimed to lead to increased (active) diffusion of all species, including solvent and reactant.8,13 (b) Reaction scheme for (i) the redox reaction between copper(II) and ascorbate ${ }^{14}$ and, (ii) the CuAAC reaction studied. (c) Representative diffusion ${ }^{1} \mathrm{H}$ NMR spectra during the reaction.

Pulsed gradient spin-echo (PGSE) or stimulated echo (PGSTE) $\mathrm{NMR}^{5}$ can simultaneously measure the selfdiffusion coefficient, $D$, and concentration of different molecular species. The measured NMR signal intensity, I, for the PGSE sequence is given by the Stejskal-Tanner equation: ${ }^{15}$

$$
I=I_{0} \exp \left[-D \gamma^{2} g^{2} \delta^{2}\left(\Delta-\frac{1}{3} \delta\right)\right]=I_{0} \exp [-D b]
$$

where $I_{0}$ is the NMR signal intensity in the absence of the applied gradient (ideally proportional to concentration), $\gamma$ is the gyromagnetic ratio of the nucleus, $g$ and $\delta$ describe the amplitude and duration of the magnetic field gradient pulse, respectively, and the delay $\Delta$ defines the timescale over which diffusion is measured. Estimates of $D$ and $I_{0}$ are determined by nonlinear regression of Eq. (1) onto the data. In dynamic systems, factors other than $g$ that influence the signal intensity during the measurement lead to processing and analysis problems. ${ }^{16}$

During chemical reactions, concentration changes lead to time-dependent $I_{0}$ values. If the changes in concentration are correlated with the order of the gradient pulse amplitudes applied in a diffusion NMR experiment, the diffusion coefficient will be miscalculated (SI-8.2, SI13). ${ }^{17} \mathrm{~A}$ less obvious factor that influences $I_{0}$ is the relaxation rate, ${ }^{18}$ i.e. changes in the longitudinal relaxation $\left(T_{1}\right)$, or transverse relaxation $\left(T_{2}\right)$. Both $T_{1}$ and $T_{2}$ are influenced by the local environment and dynamics of the nucleus being observed, and thus depend on factors like viscosity and the presence of paramagnetic species. ${ }^{18}$

The $T_{1}$ of HDO in $\mathrm{D}_{2} \mathrm{O}$ is well-known to depend on the concentration of paramagnetic ions such as copper(II) ${ }^{18}$ or gadolinium(III). ${ }^{19}$ Typical data shown in Figure 2 demonstrates that changes in very low concentrations of copper(II) ions result in significant changes in $T_{1}$ relaxation. We find the $T_{1}$ for HDO changes from $15 \pm 1 \mathrm{~s}$ in $\mathrm{D}_{2} \mathrm{O}$ (SI-5.1) to $1.2 \pm 0.1 \mathrm{~s}$ at just $1 \mathrm{mM}$ copper(II) sulfate (SI$5.3)$, consistent with earlier reports. ${ }^{18}$ This is important as very small (i.e. catalytic) concentrations of copper(II) ions will result in substantial changes in the relaxation rates of HDO and all species in solution.

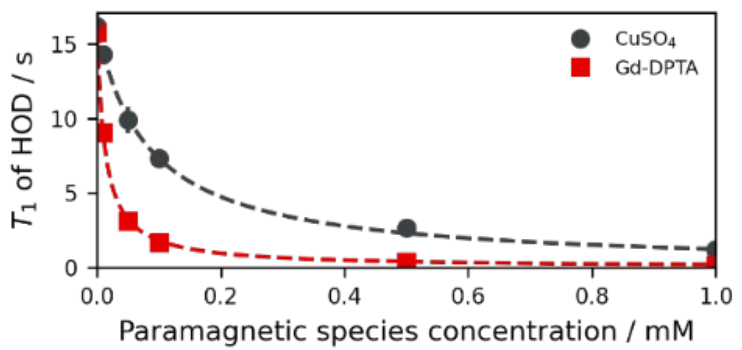

Figure 2. The dependence of relaxation constant, $T_{1}$, on the concentration of paramagnetic ions. $T_{1}$ of the residual HDO peak with varying $\mathrm{CuSO}_{4}$ or gadolinium-diethylenetriamine pentaacetic acid (Gd-DTPA) concentration, obtained by inversion recovery experiments ${ }^{1} \mathrm{H}$ NMR $500 \mathrm{MHz}, 298 \mathrm{~K}$, 12 delays ranging from $0.1 \mathrm{~ms}$ to $120 \mathrm{~s}$, depending on the expected $\left.T_{1}\right)$. Dashed lines: $T_{1}=1 /(\mathrm{a}+\mathrm{b}[\mathrm{M}])$, where $[\mathrm{M}]$ is the concentration of the paramagnetic species. ${ }^{18,20}$ Data in agreement with first reports. ${ }^{18-19}$

We now consider the $T_{1}$ of HDO during the CuAAC reaction (Figure 1b). In the initial stages of the reaction, paramagnetic copper(II) is reduced to the catalytically active copper(I), dramatically changing the $T_{1}$ of HDO. ${ }^{21}$ We find that in a $\mathrm{D}_{2} \mathrm{O}$ solution of $250 \mathrm{mM}$ azide 1 and $250 \mathrm{mM}$ alkyne 2 , the $T_{1}$ of HDO is $12 \pm 2 \mathrm{~s}$, as measured by inversion recovery experiments (SI-5.5). ${ }^{22}$ The addition of 20 $\mathrm{mM}$ copper(II) sulfate shortened the $T_{1}$ to just $69 \pm 4$ $\mathrm{ms}{ }^{23}$ a $\sim 190$-fold change (SI-5.6). ${ }^{24}$ These results indicate that the $T_{1}$ of HDO can change by two orders of magnitude as paramagnetic copper(II) is reduced to copper(I). We verified these changes by measuring the time resolved $T_{1}$ during the CuAAC reaction using saturation recovery NMR experiments (Figure 3a, SI-6). If the changing $T_{1}$ is correlated with a monotonic order of gradient amplitudes used in the diffusion experiment, ${ }^{8}$ then the diffusion coefficient of HDO appears enhanced (Figure 3d, $\square$ ). For example, in the first $10 \mathrm{~min}$ of the reaction the apparent diffusion coefficient for HDO is $2.09 \times$ $10^{-9} \mathrm{~m}^{2} \mathrm{~s}^{-1}$, compared to $1.78 \times 10^{-9} \mathrm{~m}^{2} \mathrm{~s}^{-1}$ after $40 \mathrm{~min}$. This apparent change of $\sim 17 \%$ in diffusion coefficient is not due to viscosity (SI-10) or temperature changes (SI11 ), which both vary $<2 \%$. 


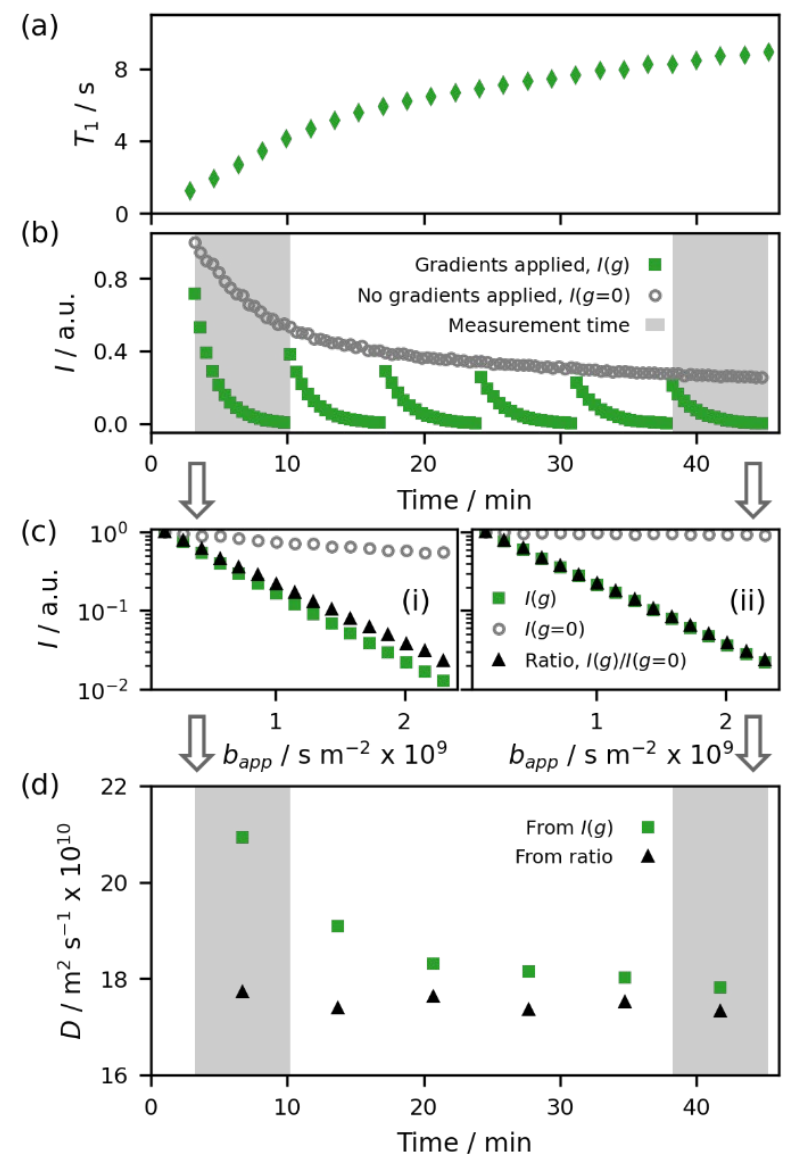

Figure 3. Effect of $T_{1}$ changes on the diffusion coefficient during the CuAAC reaction. (a) $T_{1}$ of the residual HDO peak during the $\mathrm{CuAAC}$ reaction obtained by saturation recovery. (b) Intensity from PGSTE diffusion NMR experiments with $(\square, I(g))$ and without $(O, I(g=0))$ magnetic field gradients. (c) Stejskal-Tanner plots for fitting Eq. (1) for first (i) and last (ii) diffusion experiments. $I(g=0)(0)$ shows the portion of intensity change due to $T_{1}$ changes; dividing the intensities obtained in the presence $(\square)$ and absence $(O)$ of the applied gradients $(I(g) / I(g=0), \boldsymbol{\Delta})$ corrects for the $T_{1}$ change. (d) Time-resolved diffusion coefficients calculated from the intensity $(\square)$, or the ratio $(I(g) / I(g=0), \mathbf{\Delta})$. Parameters: 250 $\mathrm{mM}$ azide 1, $250 \mathrm{mM}$ alkyne 2, $20 \mathrm{mM}$ copper(II) sulfate, 80 mM sodium ascorbate, $\mathrm{D}_{2} \mathrm{O}$; ${ }^{1} \mathrm{H}$ NMR $400 \mathrm{MHz}$, PGSTE, $\delta=$ $2.5 \mathrm{~ms}, \Delta=25 \mathrm{~ms}$, recovery delay during acquisition $\mathrm{AQ}=$ $3.15 \mathrm{s.}^{25}$

To isolate the effect of relaxation changes, we repeated the diffusion measurement during the $\mathrm{CuAAC}$ reaction with all diffusion gradient amplitudes set to zero (maintaining all other parameters). The data, shown in Figure $3 \mathrm{~b}$ without gradients $(0)$, reveal a steady decrease in the HDO signal intensity throughout the experiment. This result confirms that a proportion of the signal attenuation during this time is not due to the application of diffussion gradient pulses. The Stejskal-Tanner plots of the first and last diffusion measurement (Figure 3c) compare the signal intensities obtained with $(O)$ and without $(\square)$ the application of gradient pulses as a function of (appar- ent) $b$. The ratio of the two $(I(g) / I(g=0), \boldsymbol{\Delta})$ corresponds to the signal attenuation due to diffusion alone. Fitting this ratio (Figure 3c, $\mathbf{\Delta}$ ) results in the correct, and constant, diffusion coefficient throughout the reaction (Figure $3 \mathrm{~d}, \boldsymbol{\Delta}$, see analytical theory SI-13.3). However, this is not the most straightforward method to calculate accurate diffusion coefficients in dynamic systems.

Fortunately, it is possible to accurately determine diffusion coefficients during chemical reactions, despite changes in concentration or relaxation. This is achieved by randomly ordering (shuffling) the gradient pulse amplitudes used in the diffusion NMR experiment. Shuffling the amplitudes removes the correlation between diffusive attenuation and non-diffusion-based intensity changes (SI-8.2). ${ }^{26,10,17}$ Here we introduce a list of 500 shuffled gradients where the order of any consecutive 16 gradients has a Pearson correlation coefficient less than 0.1 (see SI-12.3). This list serves as an example to be used for any time-resolved NMR diffusion experiments. When we apply the shuffled gradient method during the CuAAC reaction we find no diffusion enhancement for any species (Figure 4a, SI-8.1), ${ }^{11 b}$ only decreased diffusion. ${ }^{11 a}$

The concentration of paramagnetic ions influences the relaxation rates of all species in solution, not just HDO. This can complicate the extraction of kinetic data, as signal integrals reflect both concentration and relaxation changes. We see this in the decreasing signal intensity of the residual HDO (Figure 3b and 4bi), despite no water being consumed in the reaction. The effect is also apparent in the non-constant sum of integrals for reactant and product signals (Figure 4ci), resulting in substantially incorrect reaction rates (Figure 4di). The signal intensity changes from dynamic relaxation constants can be counteracted by the addition of a paramagnetic relaxation agent, such as organic-soluble chromium(III) acetylacetonate ${ }^{27}$ or water-soluble gadoliniumdiethylenetriamine pentaacetic acid (Gd-DTPA). ${ }^{19-20}$

In most NMR experiments, an interscan delay of $5 \times T_{1}$ is required for complete longitudinal relaxation, which yields maximum signal. More rapid scanning will result in integrals not being strictly proportional to concentration. This will complicate analysis that requires quantitative signal intensities, including diffusion measurements. The incomplete relaxation is no problem for static diffusion NMR measurements, provided a sufficient signal-tonoise ratio is achieved. However, in dynamic systems the signal loss during the recovery delay (where the delay is $<5 \times T_{1}$ ) can become problematic. The issue is exemplified in Figure $4 \mathrm{bi}$, where $T_{1}$ of HDO increases from $70 \mathrm{~ms}$ to over $5 \mathrm{~s}$, leading to a decrease in signal intensity (here, recovery time $1.5 \mathrm{~s}$ ). A further complication in PGSTE diffusion experiments is the magnetization lost due to $T_{1}$ relaxation during the diffusion time, $\Delta$, which also reduces the signal-to-noise ratio. In cases where $\Delta>T_{1}$, the loss of magnetization during the diffusion time is very significant; this should be considered when adding a paramagnetic relaxation agent into a system. 

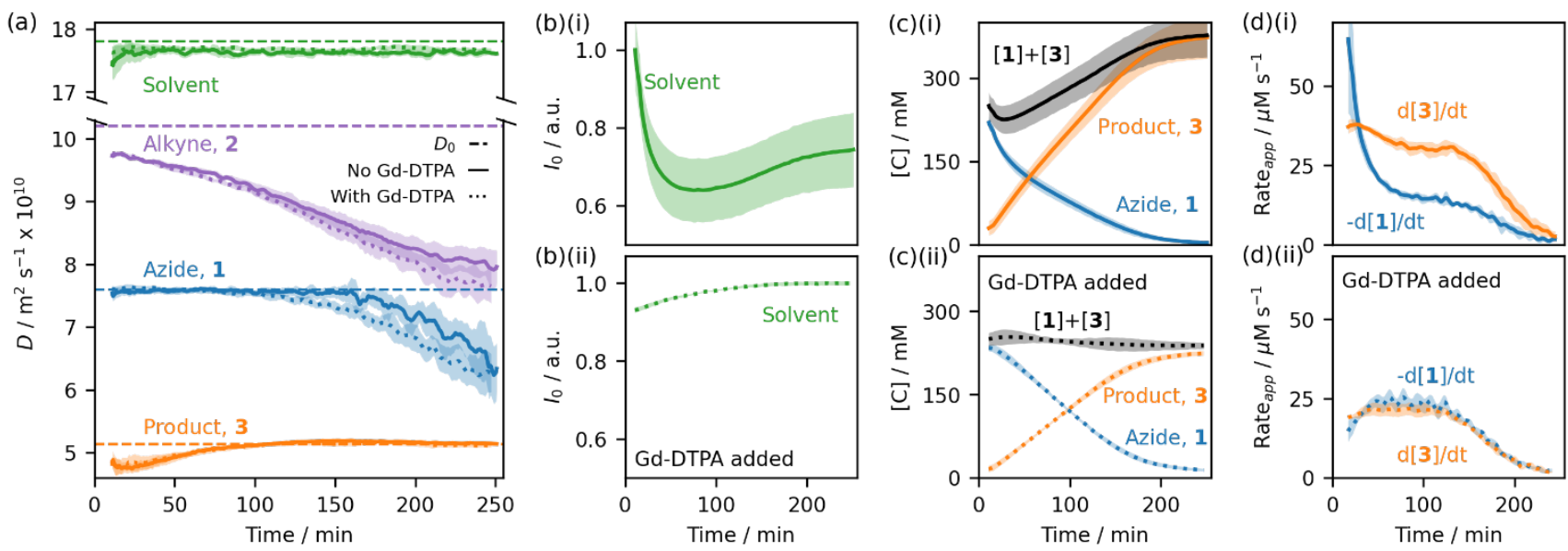

Figure 4. Addition of paramagnetic relaxation agent corrects artefacts from changing $T_{1}$. (a) Time-resolved diffusion coefficients of selected peaks during the CuACC reaction without (solid line) and with Gd-DTPA (dotted line). Dashed lines represent the control diffusion coefficients in $250 \mathrm{mM}$ azide $\mathbf{1}$, alkyne $2,80 \mathrm{mM}$ sodium ascorbate solution in $\mathrm{D}_{2} \mathrm{O}\left(D_{0}\right)$, or after the reaction for the product. (b) Solvent signal intensities $\left(I_{0}\right)$ from diffusion NMR experiments. (c) Concentrations (from $I_{0}$ ) for the $\mathrm{CH}_{2}$ group in azide 1 and its corresponding position in product 3. (d) Rates calculated from the concentrations in (c); linear fit applied over every 100 gradient slices (ca. $12 \mathrm{~min}$ ). (a-d)(i) Without and (ii) with Gd-DTPA. Parameters: $250 \mathrm{mM}$ azide 1, $250 \mathrm{mM}$ alkyne 2, $20 \mathrm{mM}$ copper(II) sulfate, $80 \mathrm{mM}$ sodium ascorbate (with $230 \mu \mathrm{M}$ Gd-DTPA), $\mathrm{D}_{2} \mathrm{O}$; ${ }^{1} \mathrm{H} 500 \mathrm{MHz}$, PGSTE, $\delta=2 \mathrm{~ms}, \Delta=50 \mathrm{~ms}$, spoiler recovery delay $=1.5 \mathrm{~s}$. Shaded area represents the standard deviation over three experiments.

For the studied CuAAC reaction, the addition of $230 \mu \mathrm{M}$ Gd-DTPA to a solution of $250 \mathrm{mM}$ of azide $\mathbf{1}$ and alkyne $\mathbf{2}$ in $\mathrm{D}_{2} \mathrm{O}$ lowered the $T_{1}$ of HDO to $270 \pm 50 \mathrm{~ms}$ (SI-5.7). Such a short $T_{1}$ satisfies the required $5 \times T_{1}$ for a relaxation delay of $1.5 \mathrm{~s}$. The addition made no substantial change to the diffusion coefficients throughout the reaction (Figure 4a). However, the intensity of the HDO signal now increases $\sim 10 \%$ throughout the reaction (Figure 5bii). This change in intensity is due to the copper catalyzed $\mathrm{H}-\mathrm{D}$ exchange between the terminal alkyne and $\mathrm{D}_{2} \mathrm{O}$, which also causes a decrease in the intensity of the terminal alkyne signal. Deuterium substitution at the terminal position is evidenced by loss of a ${ }^{4} J_{\mathrm{HH}}$ coupling $(2.3 \mathrm{~Hz})$ at the propargylic ${ }^{1} \mathrm{H}$ resonance, and deuterium coupling and intrinsic isotope shifts are observed in the ${ }^{13} \mathrm{C}$ NMR signals of the alkyne. ${ }^{28}$

The Gd-DTPA also affects the CuAAC reactants and product. For example, the $T_{1}$ of the methylene protons of azide $\mathbf{1}$ (in $250 \mathrm{mM}$ azide $\mathbf{1}$ and alkyne $\mathbf{2}$ solution) is reduced from $3.8 \pm 0.2 \mathrm{~s}$ (SI-5.5) without Gd-DTPA to 220 $\pm 100 \mathrm{~ms}$ (SI-5.7) with Gd-DTPA, which fulfills the $5 \times T_{1}$ requirement. As a result, the signal intensities more accurately represent the concentrations allowing reliable reaction kinetics to be determined (Figure 4cii, dii).

While the diffusion coefficients of the residual solvent and reducing agent (sodium ascorbate, SI-8.1) are constant over time, we notice a reproducible decrease in the diffusion coefficient of both reactants during the reaction. The decrease can be partially attributed to the overlap of these peaks with signals for the slower diffusing ascorbate which worsens as the reaction progresses (Figure 1c, SI-8.4). ${ }^{11 \mathrm{a}}$ To remove this artefact, the experiments were repeated with hydrazine monohydrate as the reducing agent instead of sodium ascorbate. As above, GdDTPA was added to allow the collection of accurate kinetic data (Figure 5). These experiments reveal a constant diffusion coefficient for azide $\mathbf{1}$, but there is still a decrease of $16 \pm 2 \%$ for the diffusion coefficient of alkyne 2 after 80 min (Figure 5a) which results from alkyne complexation to copper ions.

Measured diffusion coefficients reflect the weighted average of species that are in fast exchange on the NMR timescale. ${ }^{29}$ The fraction of the alkyne bound to copper ions increases during the reaction, and hence the ${ }^{1} \mathrm{H}$ NMR chemical shift of the terminal alkyne moves significantly downfield, consistent with copper binding (Figure 1c, SI9.2.1). ${ }^{30}$ Density functional theory (DFT) studies, following reported protocol, ${ }^{31}$ confirmed this shift is expected as the ratio of free to bound alkyne changes (SI-9.1). We also observed the alkyne ${ }^{13} \mathrm{C}$ NMR chemical shifts move downfield during the reaction (SI-9.2.2), consistent with the observation of a weighted average of copper-bound ${ }^{30}$ and free alkyne in solution. Both the ${ }^{1} \mathrm{H}$ and ${ }^{13} \mathrm{C}$ NMR chemical shifts and the diffusion coefficient of the alkyne stop changing once the reaction has reached completion. The opposite chemical shift changes were observed when we titrated free alkyne into a finished reaction, consistent with a changing average environment of free and bound alkyne (SI-9.3).

The ${ }^{1} \mathrm{H}$ and ${ }^{13} \mathrm{C}$ NMR chemical shifts of the product, especially the triazole signal, change during the reaction for the same reason: the signal is the weighted average of the copper-bound and free molecule. The proportion of the product bound to copper is highest when the product concentration is low. The bound fraction decreases as the more product is formed and the weighted average becomes closer to that of the free product. The copper ion likely binds a combination of triazole and alkyne ligands, similar to the commonly used ligand tris((1-benzyl-4triazolyl)methyl)amine (TBTA) which stabilizes copper(I) during CuAAC reactions. ${ }^{32}$ Linewidth analysis during the reaction is also consistent with the expected changing rates of exchange (SI-9.2.1). This analysis shows how a chemical reaction can be monitored using complementary NMR techniques, including diffusion NMR measurements. 

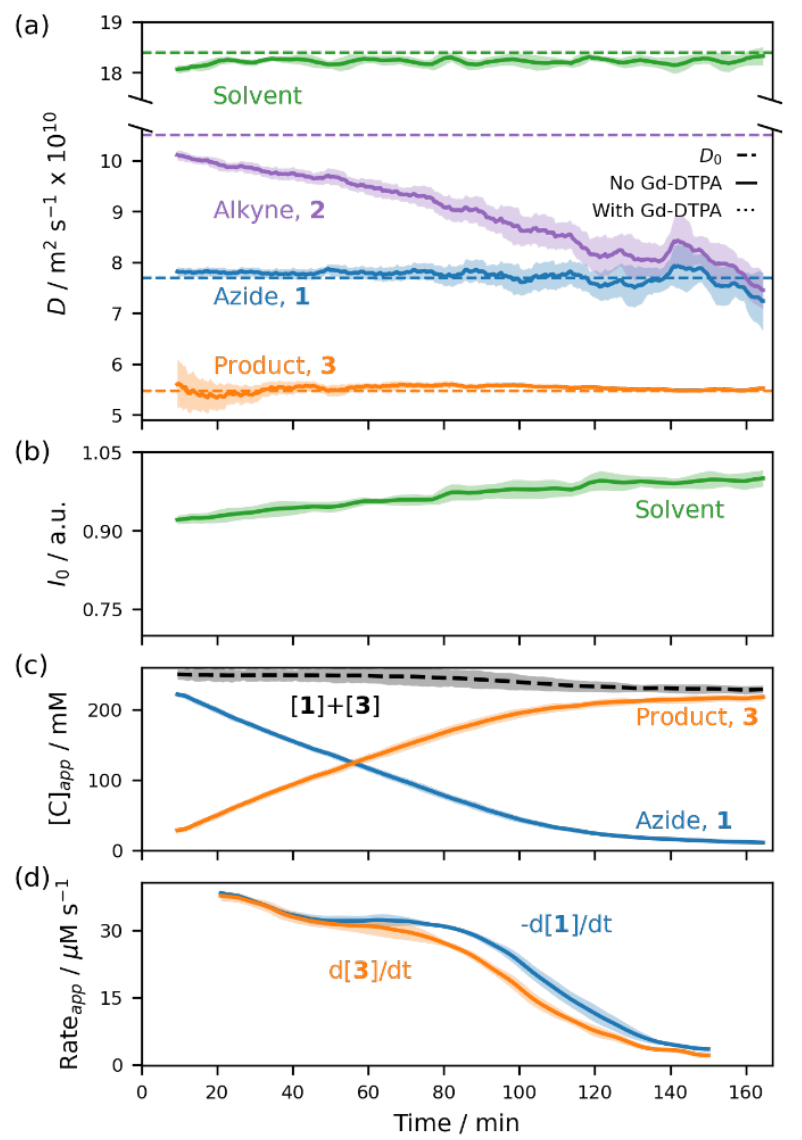

Figure 5. Alternative reducing agent removes NMR signal overlap and reveals evidence of copper complexation. (a) Time-resolved diffusion coefficients of selected peaks during the CuACC reaction; dashed line is $D_{0}$. (b) Solvent signal intensity $\left(I_{0}\right)$ extracted from diffusion NMR experiments. (c) Concentrations (from $I_{0}$ ) for the $\mathrm{CH}_{2}$ group in azide $\mathbf{1}$ and its corresponding position in product 3. (d) Rates calculated from the concentrations in (c) with linear fit applied over every 100 gradient slices (ca. $30 \mathrm{~min}$ ). Parameters: $250 \mathrm{mM}$ azide 1, $250 \mathrm{mM}$ alkyne 2, $20 \mathrm{mM} \mathrm{CuSO}_{4}, 200 \mathrm{mM}$ hydrazine monohydrate, $230 \mu \mathrm{M}$ Gd-DTPA, $\mathrm{D}_{2} \mathrm{O} ;{ }^{1} \mathrm{H} 400 \mathrm{MHz}$, PGSTE, $\delta$ $=1 \mathrm{~ms}, \Delta=10 \mathrm{~ms}$, spoiler recovery delay $=0.5 \mathrm{~s}$. Shaded areas represent the standard deviation over three experiments.

\section{Conclusion}

The addition of a paramagnetic relaxation agent assists accurate measurement of time-resolved diffusion coefficients and reaction kinetics during chemical reactions with fluctuating concentrations of paramagnetic ions. The results have been verified by three separate laboratories with different NMR spectrometers, equipped with standard or specialized probes, each using different data acquisition methods, verifying the robust nature of our protocol.

The diffusion NMR methods we present can reveal new insight into well-studied chemical reactions. Here, the observed time-dependent NMR chemical shift and diffusive behavior of the alkyne reactant is consistent with a CuAAC catalytic cycle 33 in which alkyne entry occurs through formation of a discrete copper(I)-alkyne $\pi$ com- plex. $^{34}$ To the best of our knowledge, this is the first experimental evidence for the precursor to the copper(I) acetylide, whose formation can be rate-determining for the overall cycle. ${ }^{35}$

The methods presented can be used for studying chemical systems where rates of diffusion and chemical reactions are useful parameters, such as polymerizations or supramolecular self-assemblies. Crucially, this study has shown that claims of boosted mobility during chemical reactions have no experimental basis that we could identify. Within experimental error, we show there is no diffusion enhancement at any stage of the reaction above Brownian diffusion. In stark contrast to the claim of $20 \%$ diffusion enhancement for the solvent, ${ }^{8}$ we find the diffusion coefficient is constant within $\pm 2 \%$.

\section{ASSOCIATED CONTENT}

Supporting Information. Synthetic procedures, NMR experimental data, spectra and analysis.

\section{AUTHOR INFORMATION}

\section{Corresponding Author}

* Jonathon E. Beves - School of Chemistry, UNSW Sydney, NSW 2052, Australia; ORCID: 0000-0002-5997-6580; Email: j.beves@unsw.edu.au.

* Peer Fischer - Max Planck Institute for Intelligent Systems, Heisenbergstr. 3, 70569 Stuttgart, Germany; Institute of Physical Chemistry, University of Stuttgart, Pfaffenwaldring 55, 70569 Stuttgart, Germany; ORCID: 0000-00028600-5958; E-mail: fischer@is.mpg.de.

\section{Author Contributions}

†These authors contributed equally.

\section{Funding Sources}

This work was supported by the Australian Research Council (FT170100094 to J.E.B.) and UNSW Australia (L.L.F.).

\section{ACKNOWLEDGMENT}

We acknowledge the Mark Wainwright Analytical Centre at UNSW Sydney for access to the NMR facility, Klaus Zick (Bruker BioSpin $\mathrm{GmbH}$ ) for helpful discussions on technical aspects, and Lena Steuer (Ruprecht Karl University of Heidelberg) for the suggestion to use hydrazine as reducing agent.

\section{REFERENCES}

(1) (a) Astumian, R. D. Thermodynamics and Kinetics of a Brownian Motor. Science 1997, 276, 917-922; (b) Hess, H.; Bachand, G. D.; Vogel, V. Powering Nanodevices with Biomolecular Motors. Chem.Eur. J. 2004, 10, 2110-2116; (c) Paxton, W. F.; Sundararajan, S.; Mallouk, T. E.; Sen, A. Chemical Locomotion. Angew. Chem. Int. Ed. 2006, 45, 5420-5429; (d) Goel, A.; Vogel, V. Harnessing Biological Motors to Engineer Systems for Nanoscale Transport and Assembly. Nat. Nanotechnol. 2008, 3, 465-475; (e) Golestanian, R. Enhanced Diffusion of Enzymes that Catalyze Exothermic Reactions. Phys. Rev. Lett. 2015, 115, 108102; (f) Zhang, Y.; Hess, H. Chemically-Powered Swimming and Diffusion in the Microscopic World. Nat. Rev. Chem. 2021, 5, 500-510.

(2) (a) Browne, W. R.; Feringa, B. L. Making Molecular Machines Work. Nat. Nanotechnol. 2006, 1, 25-35; (b) Kay, E. R.; Leigh, D. A.; Zerbetto, F. Synthetic Molecular Motors and Mechanical Machines. Angew. Chem. Int. Ed. 2007, 46, 72-191; (c) Abendroth, J. M.; Bushuyev, O. S.; Weiss, P. S.; Barrett, C. J. Controlling Motion at the 
Nanoscale: Rise of the Molecular Machines. ACS Nano 2015, 9, 77467768; (d) Erbas-Cakmak, S.; Leigh, D. A.; McTernan, C. T.; Nussbaumer, A. L. Artificial Molecular Machines. Chem. Rev. 2015, 115, 10081-10206; (e) Wilson, M. R.; Solà, J.; Carlone, A.; Goldup, S. M.; Lebrasseur, N.; Leigh, D. A. An Autonomous Chemically Fuelled Small-Molecule Motor. Nature 2016, 534, 235-240.

(3) Hassan, P. A.; Rana, S.; Verma, G. Making Sense of Brownian Motion: Colloid Characterization by Dynamic Light Scattering. Langmuir 2015, 31, 3-12.

(4) Visser, A. J. W. G.; Hink, M. A. New Perspectives of Fluorescence Correlation Spectroscopy. J. Fluoresc. 1999, 9, 81-87.

(5) Price, W. S. NMR Studies of Translational Motion: Principles and Applications; Cambridge University Press: Cambridge, 2009, DOI: 10.1017/CBO9780511770487.

(6) Günther, J.-P.; Majer, G.; Fischer, P. Absolute Diffusion Measurements of Active Enzyme Solutions by NMR. J. Chem. Phys. 2019, 150, 124201 .

(7) (a) Li, W.; Chung, H.; Daeffler, C.; Johnson, J. A.; Grubbs, R. H. Application of ${ }^{1} \mathrm{H}$ DOSY for Facile Measurement of Polymer Molecular Weights. Macromolecules 2012, 45, 9595-9603; (b) MacDonald, T. S. C.; Feringa, B. L.; Price, W. S.; Wezenberg, S. J.; Beves, J. E. Controlled Diffusion of Photoswitchable Receptors by Binding Antielectrostatic Hydrogen-Bonded Phosphate Oligomers. J. Am. Chem. Soc. 2020, 142, 20014-20020; (c) Zuccaccia, D.; Pinalli, R.; De Zorzi, R.; Semeraro, M.; Credi, A.; Zuccaccia, C.; Macchioni, A.; Geremia, S.; Dalcanale, E. Hierarchical Self-Assembly and Controlled Disassembly of a Cavitand-based Host-Guest Supramolecular Polymer. Polym. Chem. 2021, 12, 389-401.

(8) Wang, H.; Park, M.; Dong, R.; Kim, J.; Cho, Y.-K.; Tlusty, T.; Granick, S. Boosted Molecular Mobility during Common Chemical Reactions. Science 2020, 369, 537-541.

(9) (a) Pavlick, R. A.; Dey, K. K.; Sirjoosingh, A.; Benesi, A.; Sen, A. A Catalytically Driven Organometallic Molecular Motor. Nanoscale 2013, 5, 1301-1304; (b) Dey, K. K.; Pong, F. Y.; Breffke, J.; Pavlick, R.; Hatzakis, E.; Pacheco, C.; Sen, A. Dynamic Coupling at the Ångström Scale. Angew. Chem. Int. Ed. 2016, 55, 1113-1117.

(10) MacDonald, T. S. C.; Price, W. S.; Astumian, R. D.; Beves, J. E. Enhanced Diffusion of Molecular Catalysts is Due to Convection. Angew. Chem. Int. Ed. 2019, 58, 18864-18867.

(11) (a) Fillbrook, L. L.; Günther, J.-P.; Majer, G.; Price, W. S.; Fischer, P.; Beves, J. E. Comment on "Using NMR to Test Molecular Mobility during a Chemical Reaction". J. Phys. Chem. Lett. 2021, 12, 5932-5937; (b) Günther, J.-P.; Fillbrook, L. L.; MacDonald, T. S. C.; Majer, G.; Price, W. S.; Fischer, P.; Beves, J. E. Comment on "Boosted Molecular Mobility during Common Chemical Reactions". Science 2021, 371, eabe8322.

(12) (a) Huang, T.; Wang, H.; Granick, S. Reply to Comment on "Using NMR to Test Molecular Mobility during a Chemical Reaction". J. Phys. Chem. Lett. 2021, 12, 5744-5747; (b) Wang, H.; Park, M.; Dong, R.; Kim, J.; Cho, Y.-K.; Tlusty, T.; Granick, S. Response to Comment on "Boosted Molecular Mobility during Common Chemical Reactions". Science 2021, 371, eabe8678.

(13) Wang, H.; Huang, T.; Granick, S. Using NMR to Test Molecular Mobility during a Chemical Reaction. J. Phys. Chem. Lett. 2021, 12, 2370-2375.

(14) Du, J.; Cullen, J. J.; Buettner, G. R. Ascorbic Acid: Chemistry, Biology and the Treatment of Cancer. Biochim. Biophys. Acta 2012 1826, 443-457.

(15) Stejskal, E. O.; Tanner, J. E. Spin Diffusion Measurements: Spin Echoes in the Presence of a Time-Dependent Field Gradient. J. Chem. Phys. 1965, 42, 288-292.

(16) Equation 1 was modified to account for sine shaped gradient pulses, see Ref 5 (Price, W. S., Kuckel, P. W., Effect of Nonrectangular Field Gradient Pulses in the Stejskal and Tanner (Diffusion) Pulse Sequence, J. Magn. Reson. 1991, 94, 1, 133-139

(17) MacDonald, T. S. C.; Price, W. S.; Beves, J. E. Time-Resolved Diffusion NMR Measurements for Transient Processes. ChemPhysChem 2019, 20, 926-930.

(18) Bloembergen, N.; Purcell, E. M.; Pound, R. V. Relaxation Effects in Nuclear Magnetic Resonance Absorption. Phys. Rev. 1948, $73,679-712$

(19) Strich, G.; Hagan, P. L.; Gerber, K. H.; Slutsky, R. A. Tissue Distribution and Magnetic Resonance Spin Lattice Relaxation Effects of Gadolinium-DTPA. Radiology 1985, 154, 723-726.
(20) Heffern, M. C.; Matosziuk, L. M.; Meade, T. J. Lanthanide Probes for Bioresponsive Imaging. Chem. Rev. 2014, 114, 4496-4539.

(21) Over time some of this copper(I) will be oxidized to copper(II) due to exposure to air.

(22) Vold, R. L.; Waugh, J. S.; Klein, M. P.; Phelps, D. E. Measurement of Spin Relaxation in Complex Systems. J. Chem. Phys 1968, 48, 3831-3832.

(23) Within error of the value for $20 \mathrm{mM}$ copper sulfate in $\mathrm{D}_{2} \mathrm{O}, 69 \pm$ $1 \mathrm{~ms}$; see SI-5.3.

(24) In a $\mathrm{D}_{2} \mathrm{O}$ solution containing just $20 \mathrm{mM}$ sodium ascorbate, the $T_{1}$ value of $\mathrm{HDO}$ was $44 \pm 4 \mathrm{~s}$ (SI-5.4), illustrating significant $T_{1}$ changes other than by paramagnetic species (see Table S3 and Table S4 for full summary of $T_{1}$ constants in static solutions).

(25) We included an analytical theory to estimate the magnitude of such effects, see SI-13.3.

(26) (a) Oikonomou, M.; Asencio-Hernández, J.; Velders, A. H.; Delsuc, M.-A. Accurate DOSY Measure for Out-of-Equilibrium Systems using Permutated DOSY (p-DOSY). J. Magn. Reson. 2015, 258, 12-16; (b) Urbańczyk, M.; Bernin, D.; Czuroń, A.; Kazimierczuk, K. Monitoring Polydispersity by NMR Diffusometry with Tailored Norm Regularisation and Moving-Frame Processing. Analyst 2016, 141, 1745-1752.

(27) Gansow, O. A.; Burke, A. R.; Mar, G. N. L. A Shiftless Relaxation Reagent for Carbon-13 Magnetic Resonance of Organometallic Carbonyl Compounds. J. Chem. Soc., Chem. Commun. 1972, 10.1039/C $39720000456,456-457$.

(28) (a) Shoolery, J. N.; Johnson, L. F.; Anderson, W. A. J. Mol. Spectrosc. 1960, 5, 110-117; (b) Wigglesworth, R. D.; Raynes, W. T.; Kirpekar, S.; Oddershede, J.; Sauer, S. P. A. Nuclear Magnetic Shielding in the Acetylene Isotopomers Calculated from Correlated Shielding Surfaces. J. Chem. Phys. 2000, 112, 736-746.

(29) (a) Kärger, J. Zur Bestimmung der Diffusion in einem Zweibereichsystem mit Hilfe von gepulsten Feldgradienten. Ann. Phys. (Berl.) 1969, 479, 1-4; (b) Melchior, J.-P.; Majer, G.; Kreuer, K.-D Why do Proton Conducting Polybenzimidazole Phosphoric Acid Membranes Perform Well in High-Temperature PEM Fuel Cells? Phys. Chem. Chem. Phys. 2017, 19, 601-612; (c) Wijesekera, D.; StaitGardner, T.; Gupta, A.; Chen, J.; Zheng, G.; Torres, A. M.; Price, W. S. A Complete Derivation of the Kärger Equations for Analyzing NMR Diffusion Measurements of Exchanging Systems. Concept Magn. Reson. A 2018, 47A, e21468.

(30) (a) Reger, D. L.; Huff, M. F. (Alkyne) (2,2'-bipyridine)copper (I) Complexes. Controlled Formation of $[\mathrm{Cu} \text { (bpy)(alkyne) }]^{+}$and $\left\{\left[\mathrm{Cu}(\text { bpy) }]_{2} \text { (alkyne) }\right\}^{2+}\right.$. Organometallics 1992, 11, 69-73; (b) Oztopcu, O.; Mereiter, K.; Puchberger, M.; Kirchner, K. A. Tri- and Tetracoordinate Copper(I) Complexes Bearing Bidentate Soft/Hard SN and SeN Ligands Based on 2-aminopyridine. Dalton Trans. 2011, 40, 7008-7021; (c) Martin, C.; Sierra, M.; Alvarez, E.; Belderrain, T. R.; Perez, P. J. Hydrotris(3-mesitylpyrazolyl)borato-copper(I) Alkyne Complexes: Synthesis, Structural Characterization and Rationalization of their Activities as Alkyne Cyclopropenation Catalysts. Dalton Trans. 2012, 41, 5319-5325.

(31) Jain, R.; Bally, T.; Rablen, P. R. Calculating Accurate Proton Chemical Shifts of Organic Molecules with Density Functional Methods and Modest Basis Sets. J. Org. Chem. 2009, 74, 4017-4023.

(32) Chan, T. R.; Hilgraf, R.; Sharpless, K. B.; Fokin, V. V. Polytriazoles as Copper(I)-Stabilizing Ligands in Catalysis. Org. Lett. 2004, 6, 2853-2855.

(33) Worrell, B. T.; Malik, J. A.; Fokin, V. V. Direct Evidence of a Dinuclear Copper Intermediate in $\mathrm{Cu}(\mathrm{I})$-Catalyzed Azide-Alkyne Cycloadditions. Science 2013, 340, 457-460.

(34) For examples of static NMR diffusion studies of reactive intermediates, see: (a) Li, D.; Keresztes, I.; Hopson, R.; Williard, P. G., Characterization of Reactive Intermediates by Multinuclear DiffusionOrdered NMR Spectroscopy (DOSY). Acc. Chem. Res. 2009, 42, 27080. (b) Schlorer, N. E.; Cabrita, E. J.; Berger, S., Characterization of Reactive Intermediates by Diffusion-Ordered NMR Spectroscopy: a Snapshot of the Reaction of ${ }^{13} \mathrm{CO}_{2}$ with $\left[\mathrm{Cp}_{2} \mathrm{Zr}(\mathrm{Cl}) \mathrm{H}\right]$. Angew. Chem. Int. Ed. Engl. 2002, 41, 107-9.

(35) (a) Rodionov, V. O.; Fokin, V. V.; Finn, M. G. Mechanism of the Ligand-Free Cu-I-Catalyzed Azide-Alkyne Cycloaddition Reaction. Angew. Chem. Int. Ed. 2005, 44, 2210-2215; (b) Kuang, G. C.; Guha, P. M.; Brotherton, W. S.; Simmons, J. T.; Stankee, L. A.; Nguyen, B. T.; Clark, R. J.; Zhu, L. Experimental Investigation on the Mechanism of Chelation-Assisted, Copper(II) Acetate-Accelerated Azide-Alkyne 
Cycloaddition. J. Am. Chem. Soc. 2011, 133, 13984-14001; (c) Seath, C. P.; Burley, G. A.; Watson, A. J. Determining the Origin of RateIndependent Chemoselectivity in CuAAC Reactions: An Alkyne-
Specific Shift in Rate-Determining Step. Angew. Chem. 2017, 56, 3314 3318

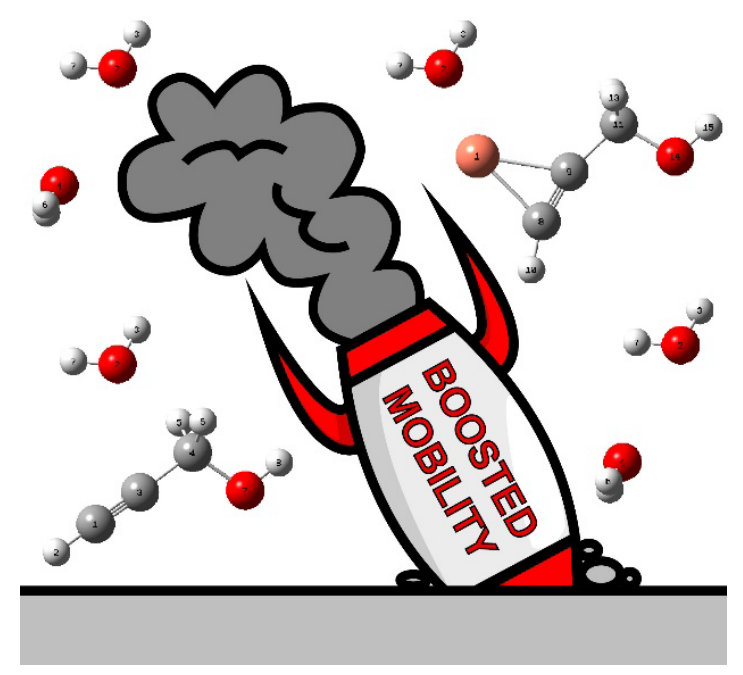

